\title{
Top quark properties
}

\author{
Javier Cuevas Maestro*† \\ U de Oviedo \\ E-mail: fjcuevas@uniovi.es
}

\begin{abstract}
An overview of recent top quark measurements in proton-proton collisions at $\sqrt{s}=7,8 \mathrm{TeV}$ in data collected with the CMS and ATLAS experiments at the LHC, using a data sample collected during the years 2011 and 2012 is presented. The results include measurements of the $t \bar{t}$ spin correlation, the top pair charge asymmetry, and the cross section of $\mathrm{t} \overline{\mathrm{t}}$ events produced in association with a $\mathrm{W}$ or a $\mathrm{Z}$ boson. The mass of the top quark is measured using different methods. Some results on the same topics are also presented in data collected by the CDF and D0 collaborations at the Tevatron collider.
\end{abstract}

XIII International Conference on Heavy Quarks and Leptons

22- 27 May, 2016

Blacksburg, Virginia, USA

\footnotetext{
* Speaker.

${ }^{\dagger}$ On behalf of the ATLAS, CMS, CDF and D0 collaborations.
} 


\section{Introduction}

The top quark is the heaviest elementary particle known, it decays before fragmenting into hadrons therefore providing a unique possibility to measure the properties of a bare quark. The large Yukawa coupling to the Higgs boson results in a large top-quark mass. For this reason, it is often believed that the top quark can play a special role in the electroweak symmetry breaking mechanism. The top quark is part of many possible beyond the Standard Model (BSM) signatures, where it can be a decay mode of, yet unknown, heavy particles. It is also foreseen that new particles can originate from top decays. Furthermore, top-quark production is an important source of background in many searches for physics BSM. The Large Hadron Collider (LHC) has operated remarkably well in 2011 and 2012, and ATLAS [1] and CMS [2] have collected large data samples of top-quark events and studies of top-quark production have been conducted in various decay channels as well as searches for deviations from the standard model (SM) predictions. Some results on the same topics are also presented in data collected by the CDF [3] and D0 [4] experiments at the Tevatron collider.

\section{Spin correlations}

Pair produced top quarks decay before their spins decorrelate, therefore their spin correlation is propagated to the $t \bar{t}$ decay products. The orientations of the top and antitop quark spins are transferred to the decay products and can be measured directly via their angular distributions. The SM predicts the $t \bar{t}$ spins to be correlated and the spin correlation strength can be defined as the asymmetry between the number of $t \bar{t}$ pairs with parallel and anti-parallel pairs. The polarizationanalyzing power of the angular distributions of charged leptons from top and antitop quark decays is effectively $100 \%$. An observable very sensitive to t $\bar{t}$ spin correlation is the azimuthal angle $\Delta \phi$ between the charged leptons.

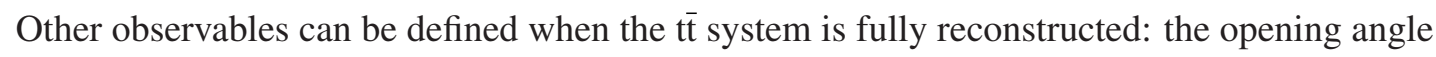
$\phi$ between the two lepton momenta measured in the rest frames of their respective parent top quark or antiquark is directly sensitive to spin correlations, as is the product of the cosines of the helicity angles of the two leptons.

ATLAS [5] has measured $\Delta \phi$ to extract the spin correlation strength

$$
A_{\text {helicity }}=\left(N_{\text {like }}-N_{\text {unlike }}\right) /\left(N_{\text {like }}+N_{\text {unlike }}\right) \text {, }
$$

where $N_{\text {like }}\left(N_{\text {unlike }}\right)$ is the number of events where the top quark and top antiquark spins are parallel (antiparallel) with respect to the spin quantization axis, respectively. This axis is chosen to be that of the helicity basis, using the direction of flight of the top quark in the center-of-mass frame of the ț̃ system.

Figure 1 (left) shows the reconstructed $\Delta \phi$ distribution for the sum of the three dilepton channels. A binned log-likelihood fit is used to extract the spin correlation from the $\Delta \phi$ distribution in data. This is done by defining a coefficient $f_{S M}$ that measures the degree of spin correlation relative to the SM prediction. The measured value of $f_{S M}$ for the combined fit is $1.20 \pm 0.05$ (stat) \pm 0.13 (syst). It agrees with the SM prediction to within two standard deviations. 
The results have been used to search for pair-produced supersymmetric top squarks decaying to top quarks and light neutralinos. Assuming $100 \%$ branching ratio for the decay $\tilde{\mathrm{t}}_{1} \rightarrow \mathrm{t} \chi_{0}^{1}$, and the production of predominantly right-handed top quarks, top squark masses between the top quark mass and $191 \mathrm{GeV}$ are excluded at 95\% C.L.

CMS [6] in addition to the inclusive measurements has determined the asymmetries differentially as a function of three variables describing the $t \bar{t}$ system in the laboratory frame: its invariant mass $M_{\mathrm{tt}}$, rapidity $y_{\mathrm{tt}}$, and transverse momentum $p_{\mathrm{tt}}^{T}$.
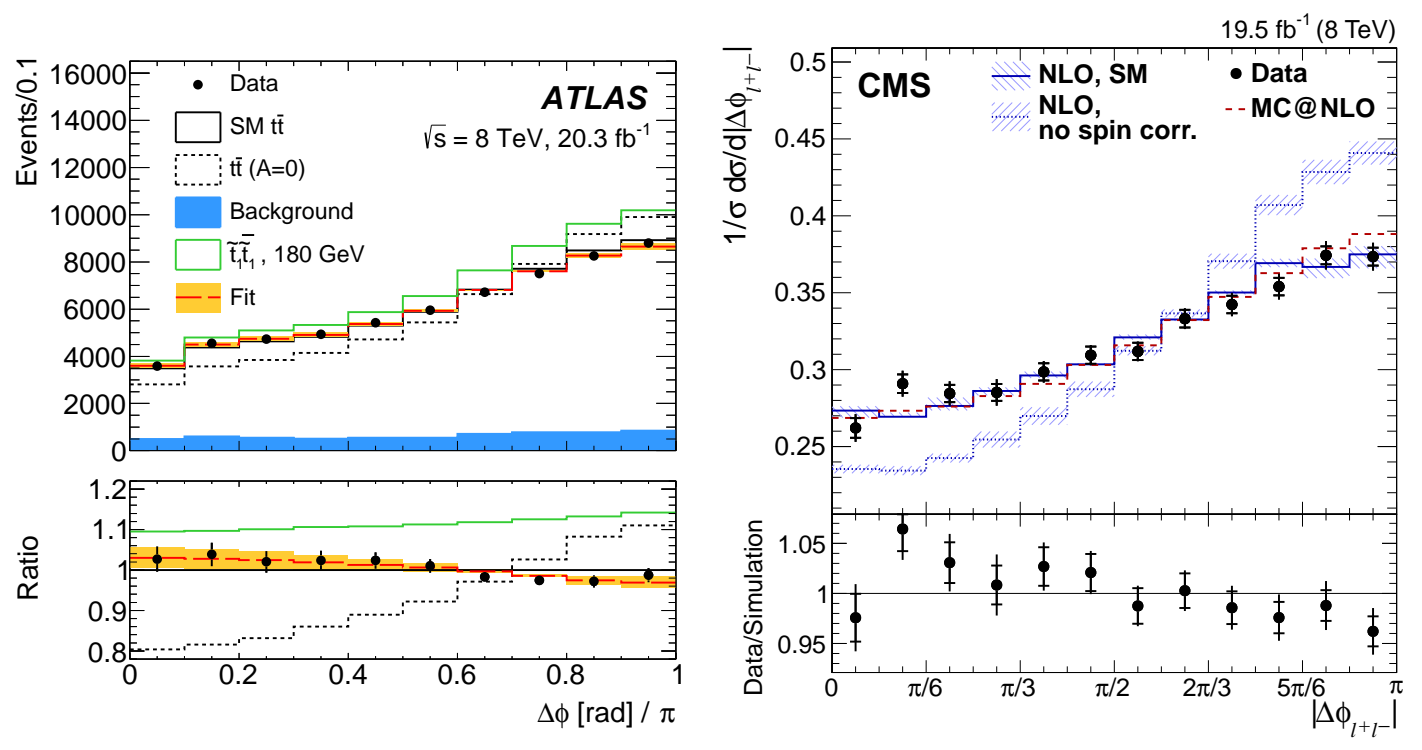

Figure 1: (left) [5] Reconstructed $\Delta \phi$ distribution for the sum of the three dilepton channels. The prediction for background (blue histogram) plus SM t⿱㇒ production (solid black histogram) and background plus $t \bar{t}$ prediction with no spin correlation (dashed black histogram) is compared to the data and to the result of the fit to the data (red dashed histogram) with the orange band representing the total systematic uncertainty on $f_{S M}$. (right) [6] Normalized differential cross section as a function of $\Delta \phi$ from data (points); parton-level predictions from MC@NLO (dashed histograms); and theoretical predictions at NLO with (SM) and without (no spin corr.) spin correlations (solid and dotted histograms, respectively).

CMS [7] tested the consistency of the spin correlation strength in top quark pair production with the SM prediction in the muon+jets final state. Using a matrix element method to discriminate between correlated and uncorrelated hypothesis, the fraction of events that show SM spin correlations is measured to be $0.72 \pm 0.08(\text { stat })_{-0.13}^{+0.15}$ (syst), representing the most precise measurement of this quantity in the muon+jets final state to date.

D0 [8] performed a measurement of the correlation between the spins of top quarks produced in proton-antiproton collisions at the Tevatron Collider at a center-of-mass energy of $1.96 \mathrm{TeV}$ applying a matrix element technique to dilepton and single-lepton+jets final states in data accumulated with the D0 detector that correspond to an integrated luminosity of $9.7 \mathrm{fb}^{-1}$. The measured value of the correlation coefficient in the off-diagonal basis, is in agreement with the Standard Model prediction.

The D0 [9] collaboration has also measured the top quark polarization in t't pair production using the full Run II data sample corresponding to $9.7 \mathrm{fb}^{-1}$ of integrated luminosity considering 
the final state containing a lepton (electron or muon) and at least three jets. The polarization is measured through the distribution of lepton angles. Three different axes are considered: the beam axis, the helicity axis, and the axis normal to the tit production plane. This is the first measurement of the transverse polarization of the top quark at a hadron collider. The observed top quark polarizations for each of the three axes are consistent with standard model predictions of nearly zero polarization. Figure 2 shows the distributions of the $\cos \theta$ distributions for data, expected backgrounds and signal templates for $P=-1,0$ and +1 .

Figure 3 shows a summary of measurements of the SM tt' spin correlation fraction $\left(f_{S M}\right)$ from different experiments and in different final states. The measurements are consistent with the SM predictions and are already systematically limited for some of the measurements.
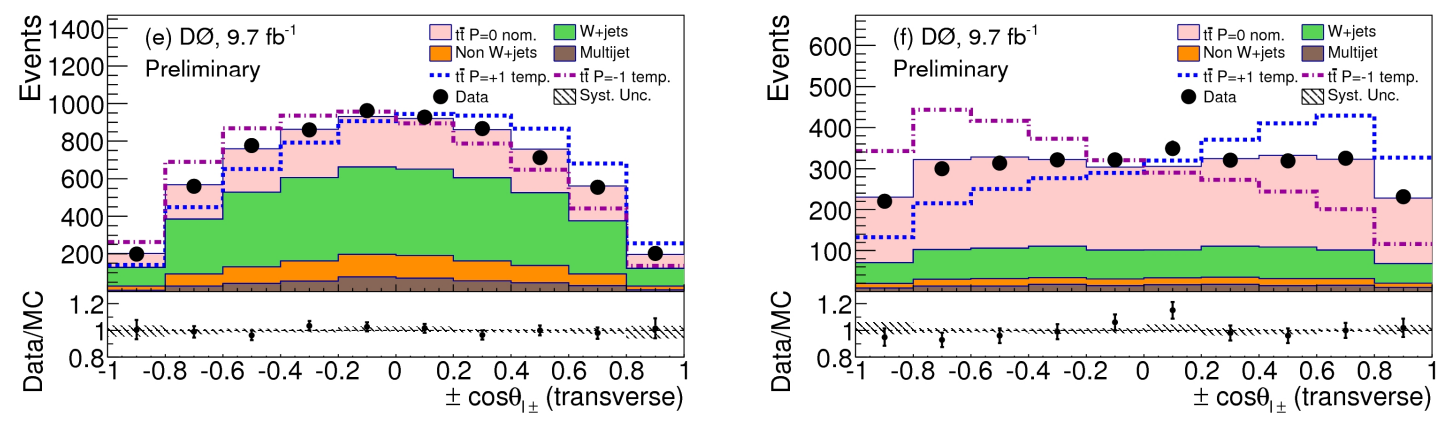

Figure 2: The combined e+jets and $\mu+\mathrm{jets} \cos \theta$ distributions for data, expected backgrounds and signal templates for $P=-1,0$ and +1 . The left panel represents the selection with exactly three jets and the right panel with four or more jets [8].

\section{Charge Asymmetry}

Top-quark pair production calculations at NLO is predicted to have a non-zero charge asymmetry from interferences between $q \bar{q}$ initiated diagrams, while the $g g$ interaction is symmetric. Measurements of forward-backward asymmetry $A_{F B}$ at the Tevatron and charge asymmetry $A_{C}$ at the LHC are complementary to evaluate new physics models. The forward-backward is characterized by the rapidity difference between top quarks and antiquarks $(\Delta y)$ and measured in the final state with two charged leptons (electrons and muons).

The LHC has a charge-symmetric, proton-proton, initial state and the top quark pairs are produced in gluon-gluon fusion and quark-antiquark annihilation processes. The valance quarks carry a larger fraction of the proton momentum which leads top quarks to be produced slightly more often in forward-backward direction and anti-top quarks more centrally. This asymmetry can be expressed as a function of the top quark and antiquark rapidities as:

$$
A_{C}=\frac{N(\Delta|y|>0)-N(\Delta|y|<0)}{N(\Delta|y|>0)+N(\Delta|y|<0)}
$$

where $\Delta|y|=\left|y_{t}\right|-\left|y_{\bar{t}}\right|$, or or by using the decay products of the top pairs as a function of the lepton pseudo-rapidities in dilepton final state:

$$
A_{C}^{l e p}=\frac{N(\Delta|\eta|>0)-N(\Delta|\eta|<0)}{N(\Delta|\eta|>0)+N(\Delta|\eta|<0)}
$$




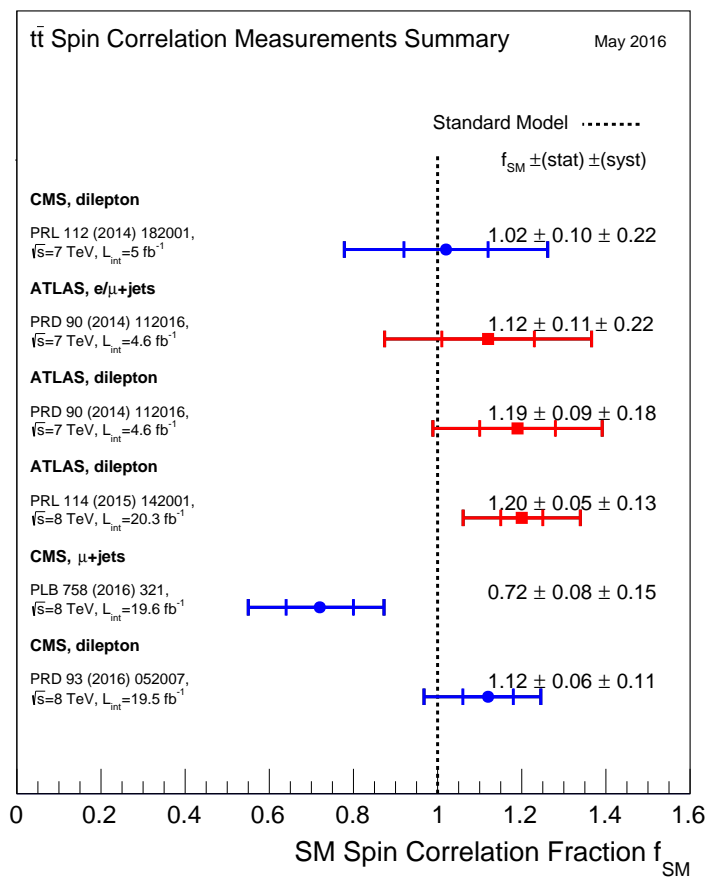

Figure 3: Summary of measurements of the SM t'tspin correlation fraction $\left(f_{S M}\right)$ from the ATLAS and CMS experiments in different final states

where $\Delta|\eta|=\left|\eta_{\ell^{+}}\right|-\left|\eta_{\ell^{-}}\right|$. SM predictions are about $1 \%$ for these observables.

The CDF [10] collaboration recently completed the legacy measurements of the forwardbackward asymmetry in the production of top-quark and antiquark pairs at the Tevatron. The inclusive asymmetry, corrected to the entire phase space at parton level, is measured to be $A_{\mathrm{tt}}^{F B}=$ $0.12 \pm 0.13$, consistent with the expectations from the standard model and previous CDF results in the final state with a single charged lepton. A measurement of the differential asymmetry as a function of $\Delta y$ was also performed. A linear fit to $A_{\mathrm{tt}}^{F B}(\Delta y)$, assuming zero asymmetry at $\Delta y=0$, yields a slope of $\alpha=0.14 \pm 0.15$, consistent with the SM prediction.

A summary of the Tevatron results and a comparison with NLO and NNLO SM predictions is shown in Figure 4.

CMS and ATLAS measured the tit charge asymmetry with different methods and in different final states at 7 and $8 \mathrm{TeV}$ centre of mass energy with the full Run I data sets. CMS [11] has measured $A_{C}$ in single-lepton events, applying an unfolding procedure to correct for detector effects, inclusively and differentially as a function of the $t \bar{t}$ system rapidity $\left|y_{\mathrm{tt}}\right|$, transverse momentum $p_{T}^{\mathrm{t} \overline{\mathrm{t}}}$ and invariant mass $m_{\mathrm{t}}$. The results are $A_{C}=[-0.35 \pm 0.72($ stat $) . \pm 0.31$ (sys.) $\left.)\right] \%$ and $A_{C}=[0.10 \pm 0.68$ (stat.) \pm 0.37 (sys.) $] \%$ for the inclusive full space and fiducial space measurements respectively. The differential measurements are also in agreement with the SM and the results are compared with predictions from an effective field theory that involves the effective axialvector coupling of the gluons. The measurement excludes new physics scales below $1.5 \mathrm{TeV}$ at the 95\% confidence level. ATLAS [12] measured the inclusive and differential t $\overline{\mathrm{t}}$ charge asymmetry $A_{C}$ in the same final state using $20.3 \mathrm{fb}^{-1}$. Three differential measurements were performed as a 
Tevatron $t \bar{t}$ asymmetry

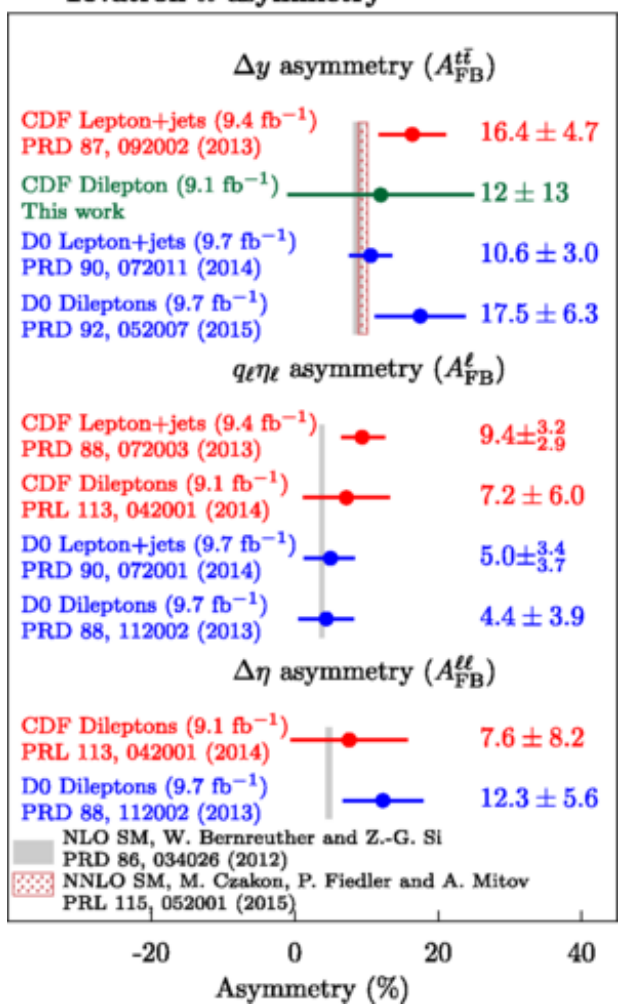

Figure 4: A comparison of all inclusive top-quark-pair forward-backward asymmetry results from the Tevatron with the NLO and NNLO SM predictions [10].

function of the invariant mass, transverse momentum and longitudinal boost of the t⿱亠幺.. A Bayesian unfolding procedure was performed to infer the asymmetry at parton level from the observed data distribution. The inclusive $\bar{t} \bar{t}$ charge asymmetry was measured to be $A_{C}=[0.9 \pm 0.5($ stat.+ syst. $)] \%$

Figure 5 shows $A_{C}$ compared to $A_{F B}$ as measured at Tevatron and predicitions given by various BSM models. The precision of the measurements allowed for the exclusion of a large phase-space of the parameters describing those BSM models.

The charge asymmetry was also measured [13] using reconstruction techniques specifically designed for the decay topology of highly boosted top quarks. The charge asymmetry in a fiducial region with large invariant mass of the top-quark pair $\left(m_{\mathrm{tt}}>0.75 \mathrm{TeV}\right)$ and an absolute rapidity difference of the top and anti-top quark candidates within $-2<\left|y_{t}\right|-\left|y_{\bar{t}}\right|<2$ was measured to be $[4.2 \pm 3.2] \%$, in agreement with the standard model prediction at NLO. Another inclusive measurement [14] was performed by CMS in the same final state with a template technique based on a parametrization of the SM. The result obtained is $A_{C}=[0.33 \pm 0.26$ (stat.) \pm 0.33 (sys.) $] \%$.

The CMS dilepton analysis [15] also includes an asymmetry measurement based on the lepton pseudo-rapidities The results are $A_{C}^{l e p}=[0.3 \pm 0.6$ (stat. $) \pm 0.3($ sys. $\left.)\right] \%$ and $A_{C}=[1.1 \pm 1.1$ (stat. $) \pm$ 0.7 (sys.)] $\%$ for the inclusive full space measurements. ATLAS [16] studied similar observables in the dilepton final state. The inclusive asymmetries are measured in the full phase space to be $A_{C}^{l e p}=0.008 \pm 0.006$ and $A_{C}^{\bar{E}} 0.021 \pm 0.016$, which are in agreement with the standard model predictions of $A_{C}^{l e p}=0.0064 \pm 0.0003$ and $A_{C}=0.0111 \pm 0.0004$. 
Figure 6 show a summary of the charge asymmetry measurements on ATLAS and CMS at 7 $\mathrm{TeV}$, both the ttbar-based and lepton-based asymmetry measurements, compared to the respective theory predictions.
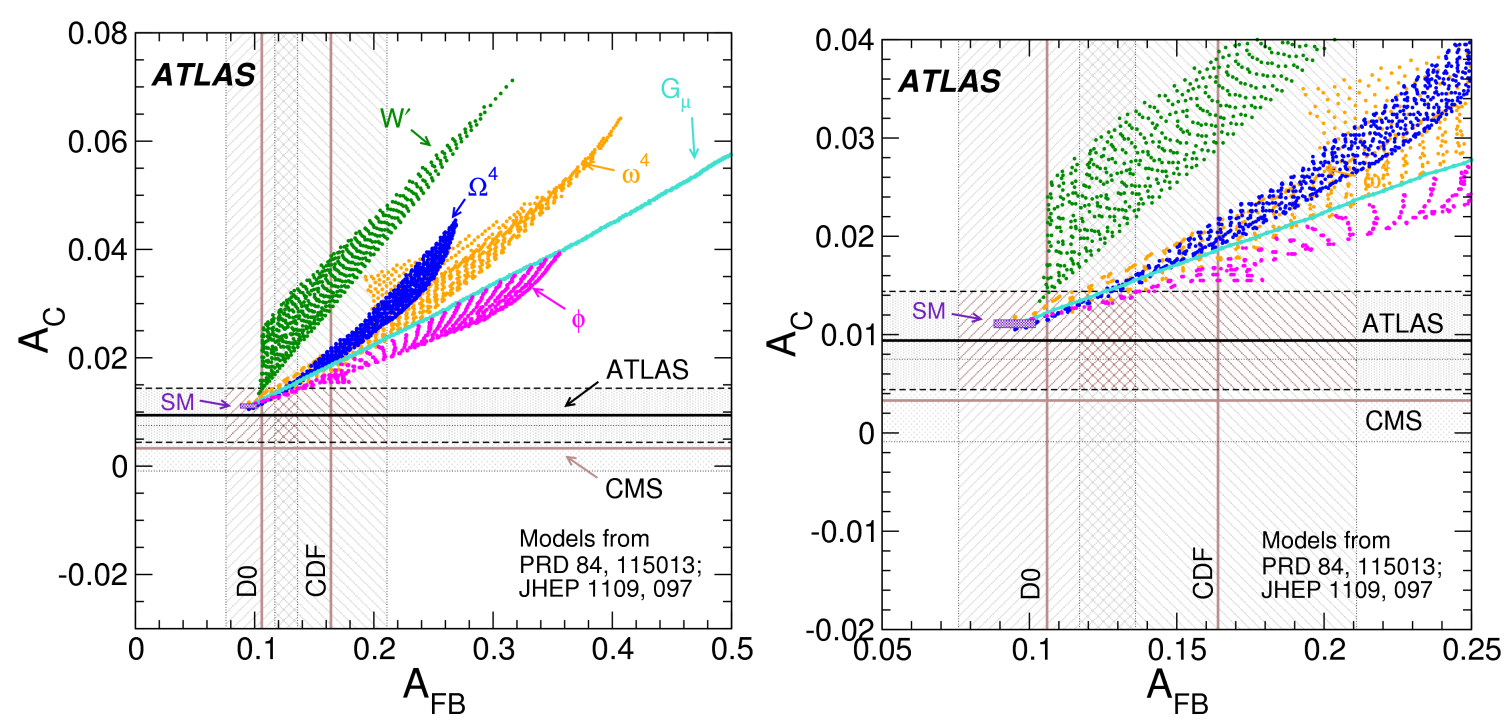

Figure 5: Measured inclusive charge asymmetries $A_{C}$ at the LHC versus forward-backward asymmetries $A_{F B}$ at Tevatron, compared with the SM predictions as well as predictions incorporating various potential BSM contributions a $W^{\prime}$ boson, a heavy axigluon $\left(G_{\mu}\right)$, a scalar isodoublet $(\Phi)$, a colour-triplet scalar $\left(\omega^{4}\right)$, and a colour-sextet scalar $\left(\Omega^{4}\right)$. The horizontal bands and lines correspond to the ATLAS and CMS measurements, while the vertical ones correspond to the CDF and D0 measurements. The figure on the right is a zoomed-in version of the figure on the left [12].

\section{Associated production of $\mathrm{t}^{\mathrm{t}}$ and bosons.}

Measurements of the $\mathrm{t} \overline{\mathrm{t}} \mathrm{Z}, \mathrm{t} \overline{\mathrm{t}} \gamma$ and $\mathrm{t} \overline{\mathrm{t}} \mathrm{H}$ processes provide a means of directly determining top quark couplings to bosons. The ttwW process is among other things, a SM source of same-sign dilepton events, which are a signature of many models of physics beyond the SM. It is also interesting per-se as it tags the process $q q \rightarrow \mathrm{tt} X$ at the LHC.

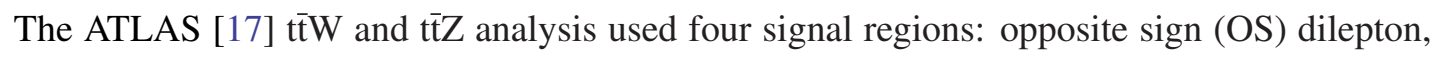
same sign (SS) dilepton, and three and four leptons. They were further split into categories depending on jet multiplicity, number of b-tagged jets and missing $E_{T}$, optimised individually to increase sensitivity. The production cross sections $\sigma_{\mathrm{t} t W}$ and $\sigma_{\mathrm{t} \mathfrak{Z} Z}$ were determined simultaneously using a binned maximum-likelihood fit over all regions and discriminant bins considered in the analysis. For both processes, the precision of the measurement is dominated by the statistical uncertainty. For the $\mathrm{tt} W$ fit, the dominant systematic uncertainty source is the modeling of fake leptons and background events with misidentified charge. For the t⿱t$Z$ fit, the dominant systematic uncertainty source is the modeling of backgrounds from simulation. The ttit $\mathrm{W}$ and $\mathrm{t} \overline{\mathrm{Z}} \mathrm{Z}$ production cross sections were measured to be $\sigma_{\mathrm{t} \tilde{W}}=369_{-91}^{+100} \mathrm{fb}$ and $\sigma_{\mathrm{ttZ}}=176_{-52}^{+58} \mathrm{fb}$. The fit to the data considering both signal processes simultaneously yields significances of $5.0 \sigma$ and $4.2 \sigma$ over the background-only hypothesis for the $\mathrm{t} \overline{\mathrm{t}} \mathrm{W}$ and $\mathrm{t} \overline{\mathrm{Z}}$ processes, respectively. 

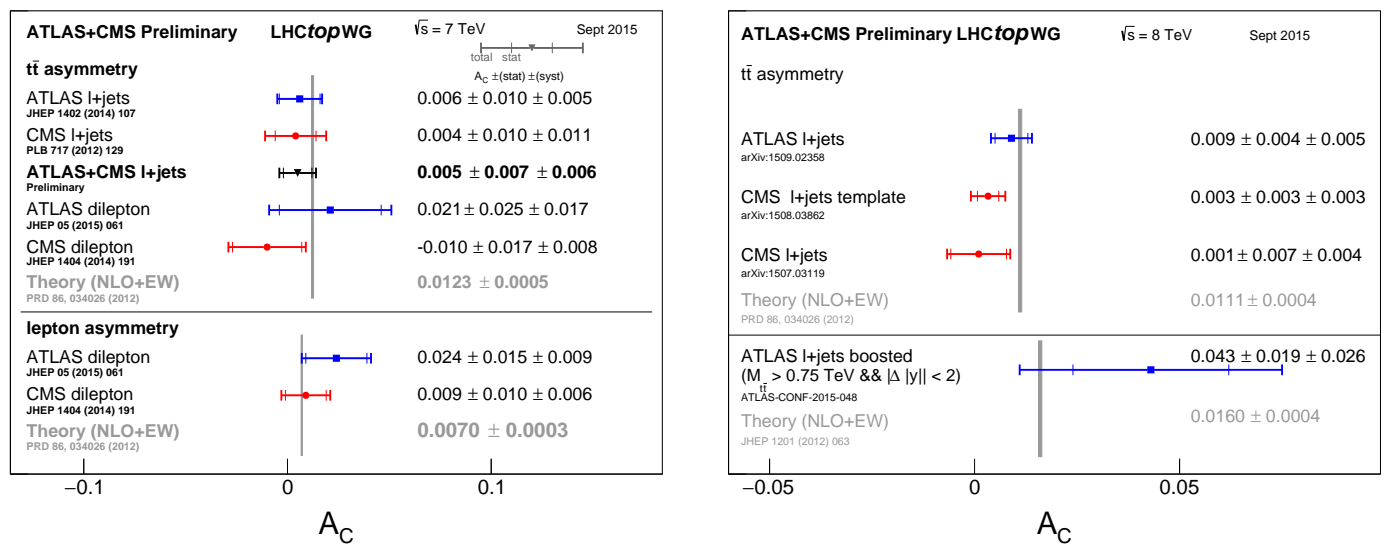

Figure 6: (left) Summary of the charge asymmetry measurements on ATLAS and CMS at $7 \mathrm{TeV}$ showing both the ttbar-based and lepton-based asymmetry measurements, compared to the respective theory predictions. (right) Summary of the charge asymmetry measurements on ATLAS and CMS at $8 \mathrm{TeV}$ showing both the inclusive measurements and the measurement using boosted events which is restricted to Mtt $>0.75$ $\mathrm{TeV}$ and $\operatorname{abs}(\operatorname{delta}(\operatorname{abs}(\mathrm{y})))<2$, compared to the respective theory predictions. The uncertainty on the theory predictions is included but not visible.

CMS [18] also performed these measurement in many channels with different numbers of leptons, jets and $\mathrm{b}$ tags, and additionally performed a full event reconstruction by matching jets and leptons to W/Z bosons and top combining into a linear discriminant and choosing the best permutation. Finally match scores were combined with kinematic quantities in BDTs. The tt̄W and $\overline{\mathrm{t}} \overline{\mathrm{Z} Z}$ production cross sections were measured to be $\sigma_{\mathrm{t} \mathrm{t} W}=382_{-102}^{+117} \mathrm{fb}$ and $\sigma_{\mathrm{t} \mathrm{ZZ}}=242_{-55}^{+65} \mathrm{fb}$, with significances of $4.8 \sigma$ and $6.4 \sigma$ over the background-only hypothesis for the $t \bar{t} \mathrm{~W}$ and $\mathrm{tt} Z$ processes, respectively. All measurements are consistent with the NLO QCD theoretical calculations.

Figure 7 shows at the left, the distribution of event yields in the analysis of the SS- $e \mu$ final state performed by ATLAS [17] and at the right the final discriminating variable in the analysis of the t⿱ẗZ three lepton final state performed by CMS [18].

\section{Top-quark mass measurements}

The top-quark mass $\left(m_{\mathrm{t}}\right)$ is an essential parameter of the standard model. It has been determined [19] with high precision at the Fermilab Tevatron to be $m_{\mathrm{t}}=174.30 \pm 0.65 \mathrm{GeV}$ from $\mathrm{t} \overline{\mathrm{t}}$ events in $\bar{p} \overline{\mathrm{p}}$ collisions.

At the CERN LHC, the ATLAS and CMS collaborations have measured the top-quark mass in all channels [21], [23], [24]. In the lepton+jets and all-hadronic final states direct reconstruction methods of the $t \bar{t}$ system were used and kinematic constrained fits performed to improve the mass resolution. In some cases the light jet energy scale was also constrained by in situ using the $\mathrm{W}$ mass. $m_{\mathrm{t}}$ is then determined with MC template fits or with event by event likelihood fits. In general the methods used were sensitive to the description of radiation and JES uncertainties. Figure 8 (left) shows the distributions after the kinematic fit of the $m_{t o p}^{\text {reco }}$ for all possible permutations.

The top-quark mass has also been measured in the dilepton final state. ATLAS [24] exploited the template method, and used the distribution of invariant masses of lepton-b-jet pairs 

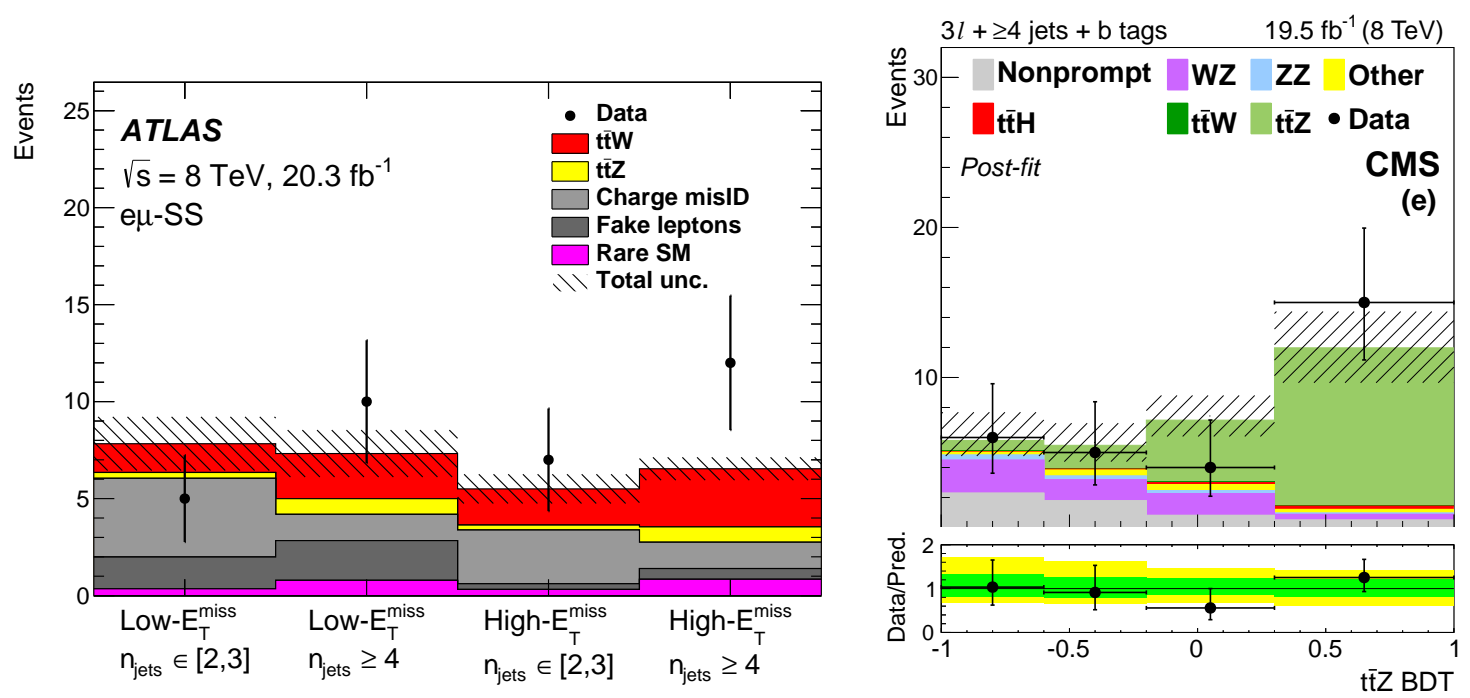

Figure 7: (left) Event yields in the ATLAS same-sign dilepton signal regions in the $e \mu$ final state [17]. (right) The final discriminant for the CMS tt̃Z channel in events with three leptons and 3 [18].

$m_{l b}^{\text {reco }}$, measuring a value of $m_{t o p}=172.99 \pm 0.41($ stat $) \pm 0.74($ syst $) \mathrm{GeV}$. The $m_{\ell b}^{\text {reco }}$ distribution in the data is shown in Figure 8 (right). CMS [21] obtained in this channel a value of $m_{\text {top }}=172.82 \pm 0.19$ (stat) \pm 1.22 (syst) $\mathrm{GeV}$, and D0 [22] $m_{\text {top }}=173.93 \pm 1.6($ stat $) \pm 0.88$ (syst) $\mathrm{GeV}$.

A summary of the direct measurements can be seen in Figure 9 (left). Both experiments have also extracted the top-quark pole mass by comparing the $t \bar{t}$ cross-section calculated at NNLO+NNLL accuracy with the measured cross-sections at 7 and $8 \mathrm{TeV}$. This is a theoretically clean method but the precision is less than what can be achieved with the direct measurements of the invariant mass of the top decay products. A summary of results is shown in Figure 9 (right) together with the measurements performed by Tevatron experiments. D0 [20] recently measures a value of $m_{\text {top }}=172.8 \pm 1.1$ (theo. $)_{-3.4}^{+3.2}($ exp. $) \mathrm{GeV}$.

\section{Summary}

The ATLAS and CMS detectors have developed a full program of measurements related to the top-quark at 7 and $8 \mathrm{TeV}$ using the data collected at both energies during the years 2011 and 2012, and previously the CDF and D0 experiments at Tevatron with the full luminosity collected until 2011. Top-quark physics is a crucial part of the current research program in High Energy Physics, key to QCD, Electro-Weak and searches for new physics. It constitutes an ideal probe to look for new physics beyond the SM with precision measurements or with direct searches for new signals. The results presented here summarises what the LHC and Tevatron experiments have measured in terms of top-quark properties. 

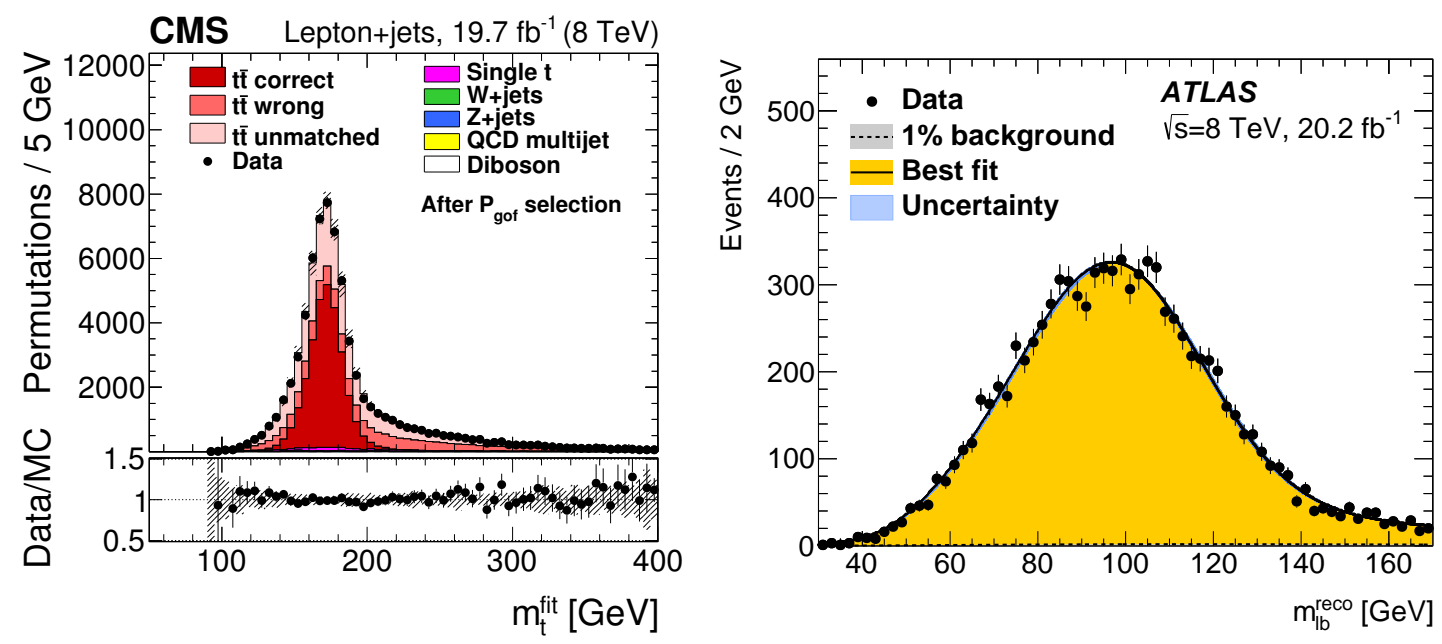

Figure 8: (left) [21] CMS fitted top quark masses after the goodness-of-fit selection in the lepton+jets final state. The vertical bars show the statistical uncertainty and the hatched bands show the statistical and systematic uncertainties added in quadrature. The lower portion of the panel shows the ratio of the yields between the collision data and the simulation. (right) [24] ATLAS $m_{\ell b}^{r e c o}$ distribution observed in data with statistical uncertainties in comparison to the prediction. The background contributions are too small to be distinguished.
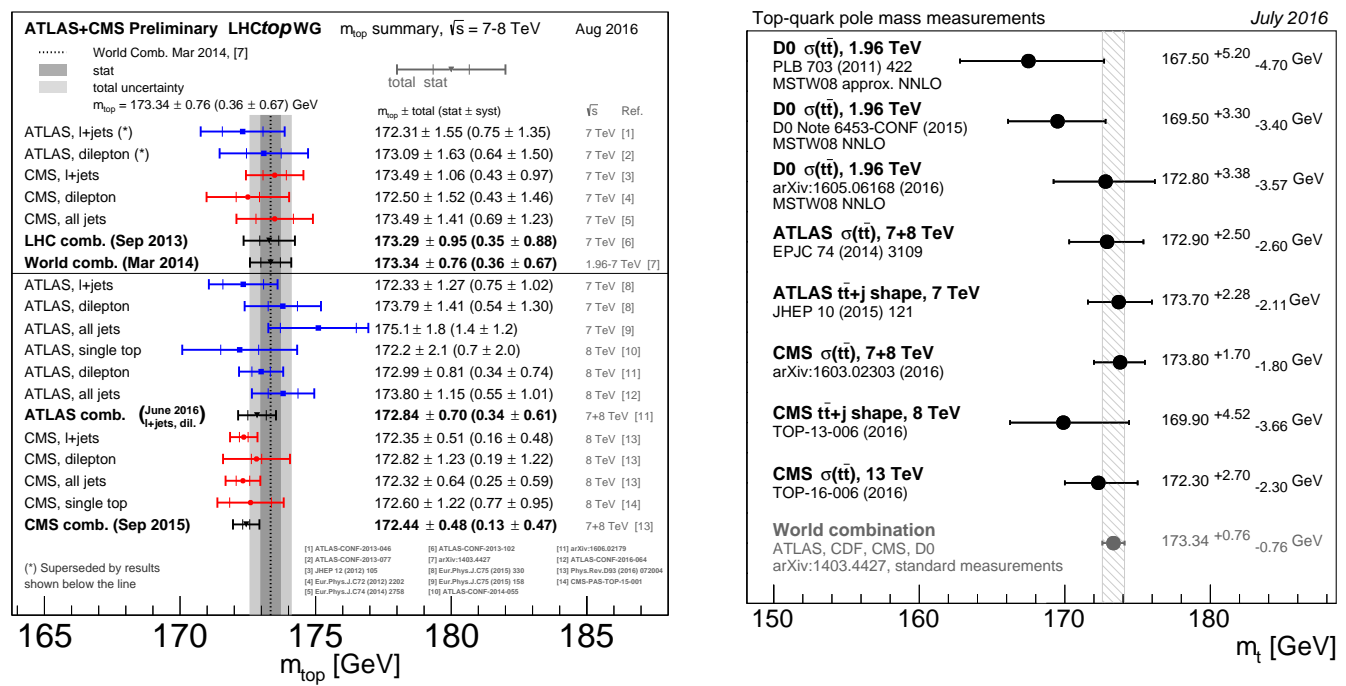

Figure 9: (left) Values of different top-quark MC mass measurements performed in ATLAS and CMS compared with the world average. (right) Summary of $m_{t o p}^{\text {pole }}$ measurements at Tevatron and LHC. 


\section{References}

[1] ATLAS collaboration, JINST 3, S08003 (2008)

[2] CMS collaboration, JINST 3, S08004 (2008)

[3] CDF Collaboration, PRD 71,052003 (2005).

[4] D0 Collaboration, NIM A 565,463 (2006).

[5] ATLAS collaboration, PRL 114, 142001 (2015)

[6] CMS collaboration, PRD 93, 052007 (2016)

[7] CMS collaboration, Phys. Lett. B 758 (2016) 321

[8] D0 collaboration, Phys. Lett. B 757 (2016) 199

[9] D0 collaboration, arxiv:1607.07627v1

[10] CDF collaboration, PRD 93, 112005 (2016)

[11] CMS collaboration, Phys. Lett. B 757 (2016) 154

[12] ATLAS collaboration, EPJC 76:87 (2016)

[13] ATLAS collaboration, Phys. Lett. B 756 (2016) 52

[14] CMS collaboration, PRD 93, 034014 (2016)

[15] CMS collaboration, Phys. Lett. B 760 (2016) 365

[16] ATLAS collaboration, arXiv:1604.05538

[17] ATLAS collaboration, JHEP11(2015)172

[18] CMS collaboration, JHEP01(2016)096

[19] CDF and D0 collaborations, arXiv:1608.01881

[20] D0 collaboration, arxiv:1605.06168

[21] CMS collaboration, PRD 93, 072004 (2016)

[22] D0 collaboration, PRD 94, 032004 (2016)

[23] ATLAS collaboration, EPJC 75:330 (2015)

[24] ATLAS collaboration, arXiv:1606.02179 